\title{
Supplementary information to Structural and Electrical Properties of Lithium Substituted Niobo Vanadate Glasses Doped with Nickel Ferrite
}

\section{This supplementary information contains the details of basic characterization of VNL glasses and Nickel Ferrite as well as tables of various parameters used to fit the Cole-Cole representation of impedance data.}

\section{S1. Physical property measurements and characterization:}

Density of the prepared glasses were determined using the Archimedes principle, to an accuracy of $\pm 0.004 \mathrm{~g} / \mathrm{cc}$. Xylene was used as an immersion liquid with a density of $0.864 \mathrm{~g} / \mathrm{cc}$ (declared by the manufacturer). A high resolution electronic weighing balance (with an accuracy of $0.0001 \mathrm{~g}$ ) was used to measure the weights of the samples in air and liquid. The molar volume was calculated using the equation $V_{m}=\frac{M}{\rho}$, where $M$ is the molecular weight of the glass sample, which was calculated as $M=\sum x_{i} w_{i}$, where $x_{i}$ and $w_{i}$ are the mole fraction and molecular weight of the component $i$ and $\rho$ is the density, respectively. The values are listed in Table 1 in the main manuscript.

The concentration of alkali ions in the general formula $x \% A_{b} B_{c}:\left(x \% \mathrm{Li}_{2} \mathrm{O}\right)$ can be calculated from $n(A)=\frac{b x N}{100 V_{m}}$, where, $x, N$ and $V_{m}$ are mole percentage, Avogadro number and molar volume, respectively. The separation between like ions was calculated using the formula $r=(1 / n)^{1 / 3}$. The distance between $\mathrm{Li}$ and $\mathrm{O}$ was calculated by assuming that $\mathrm{O}$ lies at half distance between two Li atoms. 


\section{S2 EPR of VNL glasses:}

The oxidation state of vanadium in the glass was confirmed by EPR measurement of the glass. The absence of vanadium hyperfine signature confirms that the vanadium is in $\mathrm{V}^{5+}$ state i.e., it forms a $\mathrm{VO}_{5}$ structure. Fig.S1 shows the EPR spectra of un-doped and Nickel Ferrite doped glasses. The central broad absorption is because of the cavity resonance. The central absorption at around $3360.20 \mathrm{G}$ and $3350.15 \mathrm{G}$. for VNL 15 and VNLFe 15, respectively. The sharp absorption at around 3491.09 and $1723.07 \mathrm{G}$ for VNL is the signature of DPPH which is used as reference.
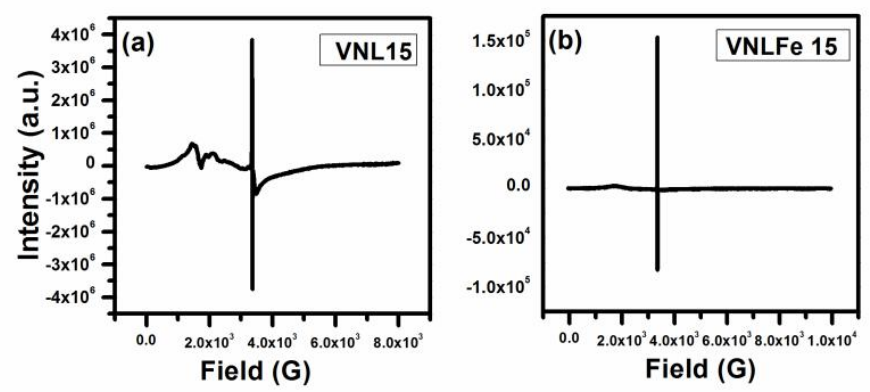

Fig. S1 (a) and (b) EPR of VNL 15 and VNLFe 15 (VNL 15 doped with Nickel Ferrite.)

\section{S3 Characterization of Nickel Ferrite:}

\section{S3.1 XRD of Nickel Ferrite (NF)}

The XRD of $\mathrm{NF}\left(\mathrm{NiFe}_{2} \mathrm{O}_{4}\right)$ is shown in Fig.S2. The crystal structure and particle size of nano

particles have been investigated using Scherer's relation using equation $D=\frac{K \lambda}{\beta \cos \theta}$ 
where $\lambda$ is the wavelength of X-ray diffraction (1.5406 $\AA$ ), $K$ is a constant taken as $0.89, \theta$ is the diffraction angle and $\beta$ is the full width half maximum (FWHM) [S1]. The crystallite size was calculated and the average crystallite size was found to be $51 \mathrm{~nm}$.

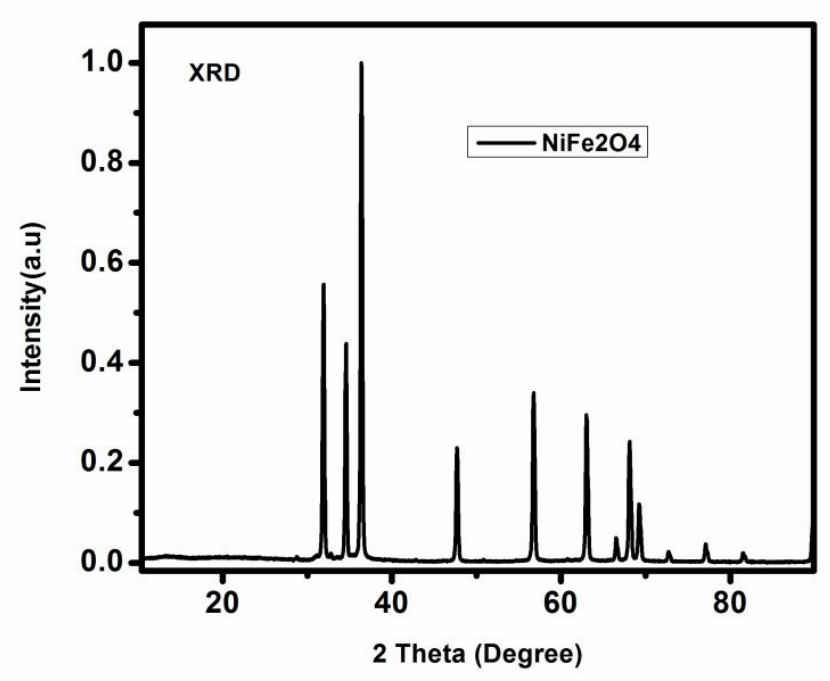

Fig. S2: XRD of Nickel Ferrite

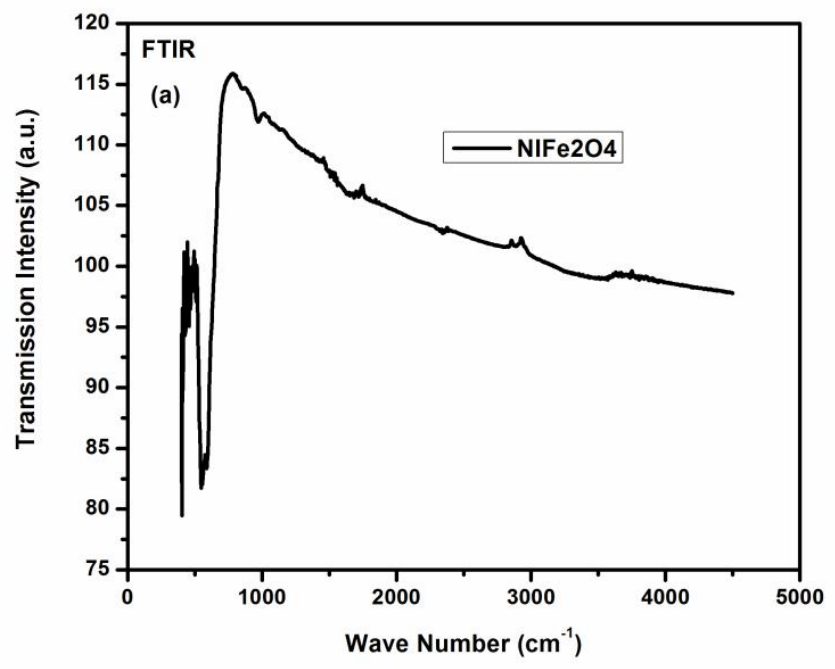

Fig. S3: FTIR of Nickel Ferrite 


\section{S3.2 SEM of Nickel Ferrite (NF)}

Microstructural information of $\mathrm{NiFe}_{2} \mathrm{O}_{4}$ (NF) was obtained using scanning electron microscopy (SEM). Field emission scanning electron microscopy was used at different magnifications to obtain a representative crystallite size and separation distance between pores in the synthesized nickel ferrite. The sample was examined at an accelerating voltage of $5 \mathrm{kV}$. The entire set-up was interfaced with a computer which enabled the direct recording of micrographs. The SEM information of nickel ferrite $\left(\mathrm{NiFe}_{2} \mathrm{O}_{4}\right)$ is shown in Fig.6. As can be seen from Fig.6 the sample is uniform, almost spherical structural morphology with narrow size distribution but with agglomeration to some extent.

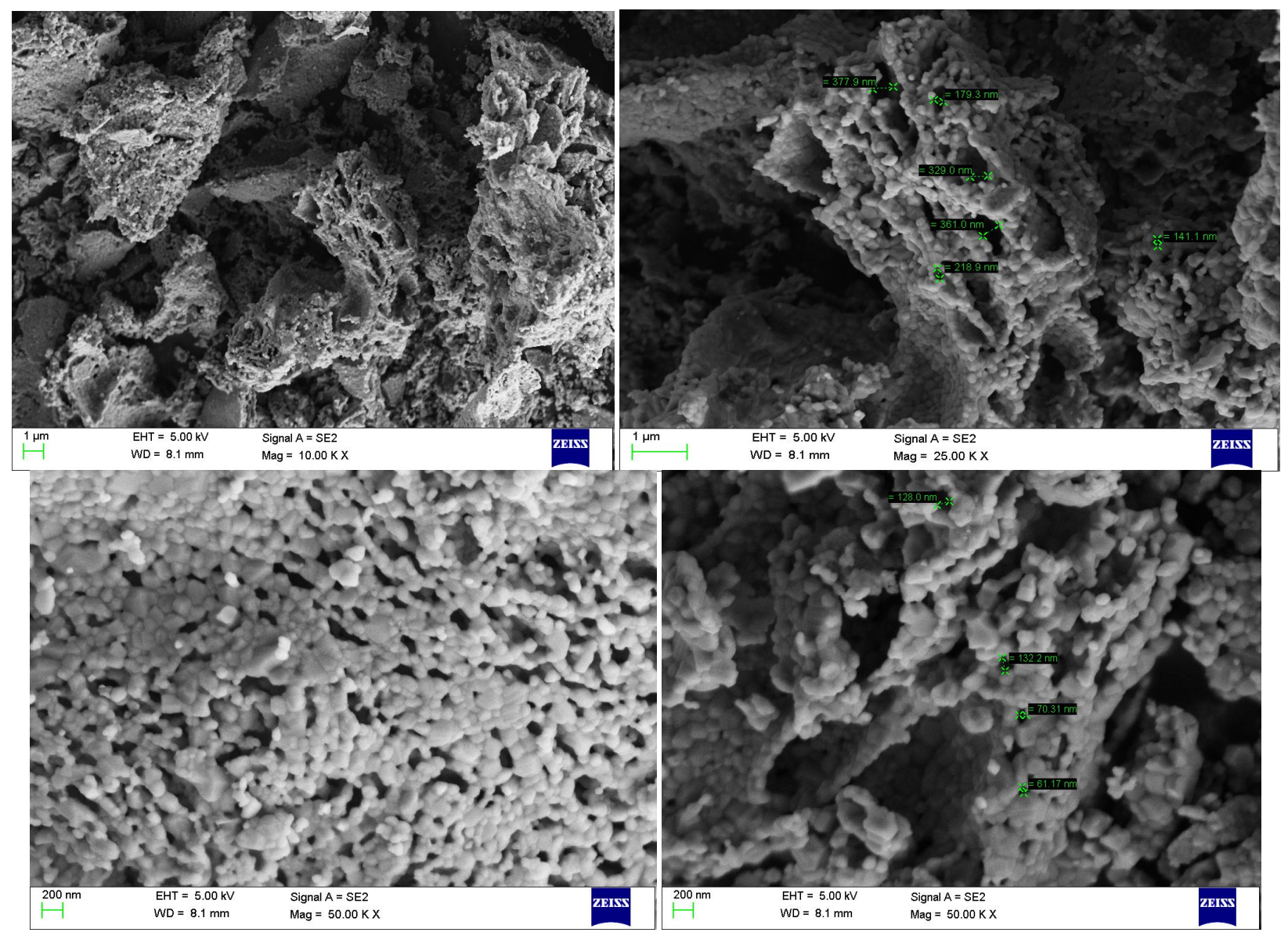


Fig. S4 : SEM Images of Nickel Ferrite $\left(\mathrm{NiFe}_{2} \mathrm{O}_{4}\right)$

S4 Tables and values of various parameters used in fitting the equivalent circuit elements to Cole-Cole plots. The meaning of the symbols and the significance are discussed in the main text.

Table S1: (a) Parameters used to fit measured Cole-Cole plot of VNL 15 using two RC circuits $\left(\mathrm{R}_{\mathrm{s}}+\mathrm{C}_{1} / \mathrm{R}_{1}+\mathrm{C}_{2} / \mathrm{R}_{2}\right)$ :Inadequate fitting

\begin{tabular}{llllll}
\hline $\begin{array}{l}\text { Temperature }\left(^{\circ} \mathbf{C}\right) \\
\text { VNL 15 }\end{array}$ & $\mathbf{R}_{\mathbf{s}}(\Omega)$ & $\mathbf{C}_{\mathbf{1}}(\mathbf{F})$ & $\mathbf{R}_{\mathbf{1}}(\mathbf{k} \Omega)$ & $\mathbf{C}_{2}(\mathbf{F})$ & $\mathbf{R}_{2}(\mathbf{k} \Omega)$ \\
\hline 120 & & & & \\
140 & 602.8 & $4.481 \times 10^{-12}$ & 89.18 & $1.076 \times 10^{-9}$ & 52.89 \\
160 & 506.4 & $4.82 \times 10^{-12}$ & 46.22 & $1.191 \times 10^{-9}$ & 34.63 \\
180 & 456.6 & $1.172 \times 10^{-9}$ & 22.43 & $4.698 \times 10^{-12}$ & 24.70 \\
200 & 467.3 & $1.137 \times 10^{-9}$ & 13.17 & $5.091 \times 10^{-12}$ & 13.61 \\
220 & 425.9 & $5.679 \times 10^{-12}$ & 7.33 & $1.02 \times 10^{-9}$ & 5.99 \\
\hline
\end{tabular}

Table S1: (b) Parameters used to fit measured Cole-Cole plot of VNL 15 using two RQ circuits $\left(\mathrm{R}_{\mathrm{s}}+\mathrm{C}_{1} / \mathrm{R}_{1}+\mathrm{C}_{2} / \mathrm{R}_{2}\right)$ : Adequate fitting 


\begin{tabular}{|c|c|c|c|c|c|c|c|}
\hline VNL 1 & & & & & & & \\
\hline 120 & $4.6 \times 10^{-24}$ & 0.915 & $16.98 \times 10^{-12}$ & 94.75 & 0.933 & $2.761 \times 10^{-9}$ & 48.06 \\
\hline 140 & -133.5 & 0.904 & $21.87 \times 10^{-12}$ & 48.75 & 0.927 & $3.021 \times 10^{-9}$ & 33.38 \\
\hline 160 & 4.39 & 0.903 & $23.68 \times 10^{-12}$ & 26.28 & 0.930 & $2.826 \times 10^{-9}$ & 22.15 \\
\hline 180 & -84.86 & 0.884 & $34.84 \times 10^{-12}$ & 1494 & 0.963 & $2.011 \times 10^{-9}$ & 13.13 \\
\hline 200 & $1.79 \times 10^{-12}$ & 0.886 & $37.65 \times 10^{-12}$ & 8.08 & 0.927 & $2.871 \times 10^{-9}$ & 6.18 \\
\hline 220 & 1.55 & 0.875 & $50.08 \times 10^{-12}$ & 4.40 & 0.934 & $2.826 \times 10^{-9}$ & 2.66 \\
\hline
\end{tabular}

Table S2: (a) Parameters used to fit measured Cole-Cole plot of VNL 20 using two RC circuits $\left(\mathrm{R}_{\mathrm{s}}+\mathrm{C}_{1} / \mathrm{R}_{1}+\mathrm{C}_{2} / \mathrm{R}_{2}\right)$ : Inadequate fitting

\begin{tabular}{llllll}
\hline $\begin{array}{l}\text { Temperature }\left({ }^{\circ} \mathbf{C}\right) \\
\text { VNL 20 }\end{array}$ & $\mathbf{R}_{\mathbf{s}}(\Omega)$ & $\mathbf{C}_{\mathbf{1}}(\mathbf{F})$ & $\mathbf{R}_{\mathbf{1}}(\mathbf{k} \Omega)$ & $\mathbf{C}_{2}(\mathbf{F})$ & $\mathbf{R}_{2}(\mathbf{k} \Omega)$ \\
\hline 120 & & & & & \\
140 & 40.46 & $3.05 \times 10^{-9}$ & $4.773 \times 10^{3}$ & $5.61 \times 10^{-12}$ & 410.75 \\
160 & 239 & $3.17 \times 10^{-9}$ & $3.511 \times 10^{3}$ & $6.13 \times 10^{-12}$ & 96.71 \\
180 & 8.41 & $6.16 \times 10^{-12}$ & 140.83 & $1.32 \times 10^{-9}$ & 261.94 \\
200 & 47.6 & $2.17 \times 10^{-9}$ & $1.267 \times 10^{3}$ & $6.42 \times 10^{-12}$ & 77.44 \\
220 & 1552 & $1.62 \times 10^{-9}$ & 280.57 & $8.04 \times 10^{-12}$ & 39.15 \\
\hline
\end{tabular}

Table S2: (b) Parameters used to fit measured Cole-Cole plot of VNL 20 using two RQ circuits $\left(\mathrm{R}_{\mathrm{s}}+\mathrm{C}_{1} / \mathrm{R}_{1}+\mathrm{C}_{2} / \mathrm{R}_{2}\right)$ : Adequate fitting

\begin{tabular}{cccccccc}
\hline $\begin{array}{c}\text { Temperature }\left(^{\circ} \mathbf{C}\right) \\
\text { VNL 20 }\end{array}$ & $\mathbf{R s}_{\mathbf{s}}(\Omega)$ & $\phi_{1}$ & $Q_{1}\left(F s^{\phi_{1}-1}\right)$ & $\mathbf{R}_{\mathbf{1}}(\mathbf{k} \Omega)$ & $\phi_{2}$ & $Q_{2}\left(F s^{\phi_{2}-1}\right)$ & $\mathbf{R}_{2}(\mathbf{k} \Omega)$ \\
\hline 120 & 170.5 & 0.955 & $2.20 \times 10^{-9}$ & 620.48 & 0.955 & $11.27 \times 10^{-12}$ & 406.74 \\
140 & 4.80 & 0.989 & $1.80 \times 10^{-9}$ & 497.79 & 0.863 & $55.39 \times 10^{-12}$ & 102.34
\end{tabular}




$\begin{array}{rrrrrrrr}160 & -421.8 & 0.940 & 2.69 \times 10^{-9} & 793.21 & 0.882 & 40.37 \times 10^{-12} & 160.31 \\ 180 & 0.58 & 0.821 & 5.46 \times 10^{-9} & 743.24 & 0.911 & 26.88 \times 10^{-12} & 77.16 \\ 200 & 3.88 & 0.906 & 4.03 \times 10^{-0} & 670.61 & 0.862 & 67.22 \times 10^{-12} & 43.04 \\ 220 & 200.7 & 0.872 & 5.24 \times 10^{-9} & 703.65 & 0.921 & 27.55 \times 10^{-12} & 21.90\end{array}$

Table S3: (a) Parameters used to fit measured Cole-Cole plot of VNL 25 using two RC circuits $\left(\mathrm{R}_{\mathrm{s}}+\mathrm{C}_{1} / \mathrm{R}_{1}+\mathrm{C}_{2} / \mathrm{R}_{2}\right)$ : Inadequate fitting

\begin{tabular}{|c|c|c|c|c|c|}
\hline $\begin{array}{l}\text { Temperature }\left({ }^{\circ} \mathrm{C}\right) \\
\text { VNL } 25\end{array}$ & $\mathbf{R}_{\mathbf{s}}(\Omega)$ & $\mathrm{C}_{1}(\mathrm{~F})$ & $R_{1}(k \Omega)$ & $C_{2}(F)$ & $\mathbf{R}_{2}(\mathrm{k} \Omega)$ \\
\hline 120 & 134.2 & $5.17 \times 10^{-12}$ & 389.33 & $12 \times 10^{-12}$ & 20.54 \\
\hline 140 & 291.4 & $0.14 \times 10^{-9}$ & $2.02 \times 10^{3}$ & $4.40 \times 10^{-12}$ & 287.08 \\
\hline 160 & 313.8 & $4.70 \times 10^{-12}$ & 146.64 & $0.97 \times 10^{-9}$ & 435.88 \\
\hline 180 & 29.7 & $4.78 \times 10^{-12}$ & 104.24 & $1.16 \times 10^{-9}$ & 417.92 \\
\hline 200 & 1627 & $1.04 \times 10^{-9}$ & 318.36 & $5.93 \times 10^{-12}$ & 46.09 \\
\hline 220 & 1947 & $1.04 \times 10^{-9}$ & 166.80 & $6.90 \times 10^{-12}$ & 29.48 \\
\hline
\end{tabular}

Table S3: (b) Parameters used to fit measured Cole-Cole plot of VNL 25 using two RQ circuits $\left(\mathrm{R}_{\mathrm{s}}+\mathrm{C}_{1} / \mathrm{R}_{1}+\mathrm{C}_{2} / \mathrm{R}_{2}\right)$ : Adequate fitting

\begin{tabular}{ccccccc}
\hline $\begin{array}{c}\text { Temperature }\left(^{\circ} \mathbf{C}\right) \\
\text { VNL 25 }\end{array}$ & $\mathbf{R}_{\mathbf{S}}(\Omega)$ & $\phi_{1}$ & $Q_{1}\left(F s^{\phi_{1}-1}\right)$ & $\mathbf{R}_{\mathbf{1}}(\mathbf{k} \Omega)$ & $\phi_{2}$ & $Q_{2}\left(F S^{\phi_{2}-1}\right)$ \\
\hline 120 & 13.84 & 0.996 & $17.06 \times 10^{-12}$ & 607.49 & 0.857 & $15.36 \times 10^{-12}$
\end{tabular}




\begin{tabular}{rccccccc}
140 & 2.14 & 0.968 & $0.72 \times 10^{-9}$ & 396.79 & 0.876 & $31.6 \times 10^{-12}$ & 360.09 \\
160 & 1.59 & 0.831 & $4.21 \times 10^{-9}$ & 829.46 & 0.898 & $24.42 \times 10^{-12}$ & 155.61 \\
180 & 155.4 & 0.963 & $1.57 \times 10^{-9}$ & 494.26 & 0.905 & $22.6 \times 10^{-12}$ & 50.75 \\
200 & 95.84 & 0.926 & $2.12 \times 10^{-9}$ & 417.06 & 0.857 & $56.04 \times 10^{-12}$ & $52.65 \times 10^{-12}$ \\
220 & $0.71 \times 10^{-6}$ & 0.789 & $8.72 \times 10^{-9}$ & 678.88 & 0.865 & & \\
\hline
\end{tabular}

Table S4: (a) Parameters used to fit measured Cole-Cole plot of VNLFe 15 using two RC circuits $\left(\mathrm{R}_{\mathrm{S}}+\mathrm{C}_{1} / \mathrm{R}_{1}+\mathrm{C}_{2} / \mathrm{R}_{2}\right)$ : Inadequate fitting

\begin{tabular}{|c|c|c|c|c|c|}
\hline $\begin{array}{l}\text { Temperature }\left({ }^{\circ} \mathrm{C}\right) \\
\text { VNLFe } 25\end{array}$ & $\mathbf{R}_{\mathbf{s}}(\Omega)$ & $\mathrm{C}_{1}(\mathrm{~F})$ & $\mathrm{R}_{1}(\mathrm{k} \Omega)$ & $\mathrm{C}_{2}(\mathrm{~F})$ & $\mathbf{R}_{\mathbf{2}}(\mathrm{k} \Omega)$ \\
\hline 120 & 3157 & $18.91 \times 10^{-12}$ & 47.95 & $2.02 \times 10^{-9}$ & 213.30 \\
\hline 140 & 497.6 & $2.18 \times 10^{-9}$ & 313.27 & $13.91 \times 10^{-12}$ & 25.54 \\
\hline 160 & 470 & $14.56 \times 10^{-12}$ & 14.18 & $2.12 \times 10^{-9}$ & 220.12 \\
\hline 180 & 647.2 & $2.05 \times 10^{-9}$ & 136.94 & $17.38 \times 10^{-12}$ & 7.80 \\
\hline 200 & 277.8 & $2.06 \times 10^{-9}$ & 162.39 & $19.76 \times 10^{-12}$ & 514 \\
\hline 220 & 349.7 & $18.33 \times 10^{-9}$ & 2.62 & $2.23 \times 10^{-9}$ & 65.12 \\
\hline 240 & 307.3 & $20.88 \mathrm{e}-12$ & 1445 & $2.82 \mathrm{e}-9$ & 8959 \\
\hline
\end{tabular}

Table S4: (b) Parameters used to fit measured Cole-Cole plot of VNLFe 15 using two RQ circuits $\left(\mathrm{R}_{\mathrm{s}}+\mathrm{C}_{1} / \mathrm{R}_{1}+\mathrm{C}_{2} / \mathrm{R}_{2}\right)$ : Adequate fitting

\begin{tabular}{ccccccc}
\hline $\begin{array}{c}\text { Temperature }\left(^{\circ} \mathbf{C}\right) \\
\text { VNLFe 15 }\end{array}$ & $\mathbf{R}_{\mathbf{S}}(\Omega)$ & $\phi_{1}$ & $Q_{1}\left(F s^{\phi_{1}-1}\right)$ & $\mathbf{R}_{\mathbf{1}}(\mathbf{k} \Omega)$ & $\phi_{2}$ & $Q_{2}\left(F S^{\phi_{2}-1}\right)$ \\
\hline 120 & 26.31 & 0.874 & $6.56 \times 10^{-9}$ & 406.12 & 0.810 & $0.27 \times 10^{-9}$
\end{tabular}




$\begin{array}{llllllll}140 & 23.01 & 0.888 & 6.20 \times 10^{-9} & 924.94 & 0.809 & 0.30 \times 10^{-9} & 29.41 \\ 160 & 100.5 & 0.872 & 7.14 \times 10^{-9} & 683.73 & 0.826 & 0.25 \times 10^{-9} & \\ 180 & 744.9 & 0.902 & 5.26 \times 10^{-9} & 209.90 & 0.991 & 21.26 \times 10^{-12} & 4.53 \\ 200 & 370.6 & 0.910 & 4.99 \times 10^{-9} & 213.65 & 0.930 & 55.4 \times 10^{-12} & 3.14 \\ 220 & 60.14 & 0.956 & 3.61 \times 10^{-9} & 68.46 & 0.775 & 0.74 \times 10^{-9} & 0.84 \times 10^{-9} \\ 240 & 9.72 \times 10^{-12} & 0.919 & 7.31 \times 10^{-9} & 9.92 & 0.767 & & \end{array}$

Table S5: (a) Parameters used to fit measured Cole-Cole plot of VNLFe 20 using two RC circuits $\left(\mathrm{R}_{\mathrm{s}}+\mathrm{C}_{1} / \mathrm{R}_{1}+\mathrm{C}_{2} / \mathrm{R}_{2}\right)$ : Inadequate fitting

\begin{tabular}{|c|c|c|c|c|c|}
\hline $\begin{array}{l}\text { Temperature }\left({ }^{\circ} \mathrm{C}\right) \\
\text { VNLFe } 20\end{array}$ & $\mathbf{R}_{\mathbf{s}}(\Omega)$ & $C_{1}(F)$ & $\mathbf{R}_{1}(\mathrm{k} \Omega)$ & $\mathrm{C}_{2}(\mathrm{~F})$ & $\mathbf{R}_{2}(\mathrm{k} \Omega)$ \\
\hline 120 & 107.8 & $9.98 \times 10^{-12}$ & 59.0 & $5.67 \times 10^{-12}$ & 900.15 \\
\hline 140 & 390.6 & $1.36 \times 10^{-9}$ & $1.44 \times 10^{3}$ & $4.26 \times 10^{-12}$ & 465.53 \\
\hline 160 & 145.2 & $1.07 \times 10^{-9}$ & $1.86 \times 10^{3}$ & $4.62 \times 10^{-12}$ & 231.20 \\
\hline 180 & 161 & $1.05 \times 10^{-9}$ & 280.81 & $4.55 \times 10^{-12}$ & 109.94 \\
\hline 200 & 1180 & $1.19 \times 10^{-9}$ & 297.87 & $5.18 \times 10^{-12}$ & 57.63 \\
\hline 220 & 588.2 & $1.18 \times 10^{-9}$ & 194. 94 & $5.21 \times 10^{-12}$ & 31.36 \\
\hline 240 & 541.7 & $5.59 \times 10^{-12}$ & 17.32 & $1.43 \times 10^{-9}$ & 90.94 \\
\hline
\end{tabular}

Table S5: (b) Parameters used to fit measured Cole-Cole plot of VNLFe 20 using two RQ circuits $\left(\mathrm{R}_{\mathrm{S}}+\mathrm{C}_{1} / \mathrm{R}_{1}+\mathrm{C}_{2} / \mathrm{R}_{2}\right)$ : Adequate fitting

\begin{tabular}{lllllll}
\hline $\begin{array}{c}\text { Temperature }\left({ }^{\circ} \mathbf{C}\right) \\
\text { VNLFe 20 }\end{array}$ & $\mathbf{R}_{\mathbf{s}}(\Omega)$ & $\phi_{1}$ & $Q_{1}\left(F s^{\phi_{1}-1}\right)$ & $\mathbf{R}_{\mathbf{1}}(\mathbf{k} \Omega)$ & $\phi_{2}$ & $Q_{2}\left(F S^{\phi_{2}-1}\right)$ \\
\hline
\end{tabular}




\begin{tabular}{|c|c|c|c|c|c|c|c|}
\hline 120 & 8.97 & 0.970 & $8.10 \times 10^{-12}$ & 927.07 & 0.997 & $14.52 \times 10^{-12}$ & 53.25 \\
\hline 140 & 32.4 & 0.805 & $4.12 \times 10^{-9}$ & 572.44 & 0.911 & $17.33 \times 10^{-12}$ & 451.06 \\
\hline 160 & 168.1 & 0.968 & $1.48 \times 10^{-12}$ & 829.55 & 0.942 & $11.44 \times 10^{-12}$ & 238.23 \\
\hline 180 & 286.4 & 0.967 & $1.63 \times 10^{-9}$ & 934.64 & 0.955 & $9.42 \times 10^{-12}$ & 117.28 \\
\hline 200 & 107 & 0.956 & $1.84 \times 10^{-9}$ & 538.87 & 0.905 & $23.04 \times 10^{-12}$ & 61.93 \\
\hline 220 & -510 & 0.947 & $2.08 \times 10^{-9}$ & 329.24 & 0.841 & $68.61 \times 10^{-12}$ & 35.41 \\
\hline 240 & 556.3 & 0.923 & $3.17 \times 10^{-9}$ & 137.16 & 0.989 & $6.81 \times 10^{-12}$ & 17.18 \\
\hline
\end{tabular}

Table S6: (a) Parameters used to fit measured Cole-Cole plot of VNLFe 25 using two RC circuits $\left(\mathrm{R}_{\mathrm{S}}+\mathrm{C}_{1} / \mathrm{R}_{1}+\mathrm{C}_{2} / \mathrm{R}_{2}\right)$ : Inadequate fitting

\begin{tabular}{|c|c|c|c|c|c|}
\hline $\begin{array}{l}\text { Temperature }\left({ }^{\circ} \mathrm{C}\right) \\
\text { VNLFe } 25\end{array}$ & $R_{s}(\Omega)$ & $\mathrm{C}_{1}(\mathrm{~F})$ & $R_{1}(k \Omega)$ & $\mathrm{C}_{2}(\mathrm{~F})$ & $\mathbf{R}_{2}(\mathrm{k} \Omega)$ \\
\hline 120 & 566.9 & $30.69 \times 10^{-12}$ & 55.94 & $1.31 \times 10^{-9}$ & 598.90 \\
\hline 140 & 175.1 & $3.81 \times 10^{-9}$ & 890.23 & $5.03 \times 10^{-12}$ & 295.01 \\
\hline 160 & 378.4 & $5.11 \times 10^{-12}$ & 135.03 & $1.46 \times 10^{-9}$ & 103.99 \\
\hline 180 & 538.5 & $2.30 \times 10^{-9}$ & 130.35 & $5.40 \times 10^{-12}$ & 69.35 \\
\hline 200 & 415 & $1.01 \times 10^{-9}$ & $1.25 \times 10^{6}$ & $5.57 \times 10^{-12}$ & 33.33 \\
\hline 220 & 569.7 & $1.05 \times 10^{-9}$ & 490.88 & $6.26 \times 10^{-12}$ & 16.29 \\
\hline 240 & 572.9 & $7.62 \times 10^{-12}$ & 7.60 & $1.16 \times 10^{-9}$ & 73.57 \\
\hline
\end{tabular}

Table S6: (b) Parameters used to fit measured Cole-Cole plot of VNLFe 25 using two RQ circuits $\left(\mathrm{R}_{\mathrm{s}}+\mathrm{C}_{1} / \mathrm{R}_{1}+\mathrm{C}_{2} / \mathrm{R}_{2}\right)$ : Adequate fitting 


\begin{tabular}{|c|c|c|c|c|c|c|c|}
\hline $\begin{array}{c}\text { Temperature }\left({ }^{\circ} \mathrm{C}\right) \\
\text { VNLFe } 25\end{array}$ & $\mathbf{R}_{\mathrm{S}}(\Omega)$ & $\phi_{1}$ & $Q_{1}\left(F S^{\phi_{1}-1}\right)$ & $\mathbf{R}_{1}(\mathrm{k} \Omega)$ & $\phi_{2}$ & $Q_{2}\left(F s^{\phi_{2}-1}\right)$ & $\mathbf{R}_{2}(\mathbf{k} \Omega)$ \\
\hline 120 & 168.3 & 0.920 & $4.10 \times 10^{-9}$ & 526.23 & 0.787 & $0.72 \times 10^{-9}$ & 74.69 \\
\hline 140 & 3.15 & 0.754 & $36.16 \times 10^{-9}$ & 787.18 & 0.888 & $28.96 \times 10^{-9}$ & 317.01 \\
\hline 160 & 0.04 & 0.930 & $4.27 \times 10^{-9}$ & 245.73 & 0.886 & $32.15 \times 10^{-12}$ & 154.74 \\
\hline 180 & -287.8 & 0.705 & $41.75 \times 10^{-9}$ & 636.79 & 0.883 & $35.75 \times 10^{-12}$ & 72.99 \\
\hline 200 & 173.1 & 0.999 & $0.93 \times 10^{-9}$ & 702.22 & 0.893 & $33.12 \times 10^{-12}$ & 35.94 \\
\hline 220 & 4.85 & 0.937 & $1.93 \times 10^{-9}$ & 749. 53 & 0.856 & $68.68 \times 10^{-12}$ & 17.79 \\
\hline 240 & 440.8 & 0.907 & $3.15 \times 10^{-9}$ & 112.85 & 0.967 & $13.06 \times 10^{-12}$ & 7.56 \\
\hline
\end{tabular}

The range of the variation of these values are summarized and presented in main text as Table 2 . 